\title{
Stability and Hopf Bifurcation of a Predator-Prey Model with Distributed Delays and Competition Term
}

\author{
Lv-Zhou Zheng \\ College of Mathematics and Statistics, Hubei Normal University, Huangshi 435002, China \\ Correspondence should be addressed to Lv-Zhou Zheng; 392190186@qq.com
}

Received 31 March 2014; Accepted 12 April 2014; Published 27 April 2014

Academic Editor: Ligang Wu

Copyright (C) $2014 \mathrm{Lv}$-Zhou Zheng. This is an open access article distributed under the Creative Commons Attribution License, which permits unrestricted use, distribution, and reproduction in any medium, provided the original work is properly cited.

A class of predator-prey system with distributed delays and competition term is considered. By considering the time delay as bifurcation parameter, we analyze the stability and the Hopf bifurcation of the predator-prey system. According to the theorem of Hopf bifurcation, some sufficient conditions are obtained for the local stability of the positive equilibrium point.

\section{Introduction}

In general, delay differential equations display more complicated dynamics than ordinary differential equations as a time delay could bring a switch in the stability of equilibria and induce various oscillations and periodic solutions. Neural Networks with time delay or distributed delays have been investigated by some researchers [1-4]. Different from [14], distributed delays are introduced into a Lotka-Volterra predator-prey system. Lotka-Volterra predator-prey system is always the hot question in mathematical ecology, and many scholars have made deep and broad study in this area [5-10]. May [5] first proposed a model describing a predator-prey system with delay:

$$
\begin{aligned}
& \dot{x}(t)=x(t)\left[r_{1}-a_{11} x(t-\tau)-a_{12} y(t)\right], \\
& \dot{y}(t)=y(t)\left[-r_{2}-a_{21} x(t)-a_{22} y(t)\right],
\end{aligned}
$$

where $x(t), y(t)$ denote the densities of prey and predator at time $t$, respectively, $\tau>0$ denotes growth time of prey, $r_{1}>0$, $r_{2}>0$ denote the growth rates of prey and predator at time $t$, respectively, $a_{i i}>0(i=1,2)$ denote the inner impact factors in two populations, respectively, and $a_{i j}>0(i \neq j)$ denote the mutual impact factors in two populations, respectively.

Recently, Song and Wei [9] developed a Logistic predatorprey model with discrete and distributed delays:

$$
\dot{x}(t)=r x(t)\left[1-a_{1} x(t-\tau)-a_{2} \int_{-\infty}^{t} f(t-s) x(s) d s\right],
$$

where $r, \tau, a_{1}$, and $a_{2}$ are positive constants and $f(t)$ is the kernel function satisfying $f(t) \geq 0(t \geq 0)$ and $\int_{0}^{\infty} f(t) d t=$ 1.

In nature, living space and resources are limited. Therefore it is advisable to study a predator-prey model with competition term. The competition term describes the competitive interactions between the two species. Associated with these works in $[5,9,10]$, in this paper, a predator-prey model with distributed delays and competition term is considered.

$$
\begin{gathered}
\dot{\overline{\bar{x}}}(t)=\overline{\bar{x}}(t)\left[r-r_{1} \overline{\bar{x}}(t-\tau)-r_{2} \int_{-\infty}^{t} f(t-s) \overline{\bar{x}}(s) d s\right. \\
\left.-a_{1} \overline{\bar{y}}(t-\tau)\right], \\
\dot{\overline{\bar{y}}}(t)=\overline{\bar{y}}(t)\left[-r_{3}+a_{2} \overline{\bar{x}}(t-\tau)-r_{4} \overline{\bar{y}}(t-\tau)\right] \\
-c \overline{\bar{y}}(t) \overline{\bar{y}}^{2}(t-\tau) .
\end{gathered}
$$

Obviously, system (3) improves and contains most of existing models. For (3), we define the kernel function

$$
f(t)=\sigma e^{-\sigma t}, \quad \sigma>0 .
$$

Denote

$$
\overline{\bar{u}}(t)=\int_{-\infty}^{t} \sigma e^{-\sigma(t-s)} \overline{\bar{x}}(s) d s
$$


Then we have

$$
\begin{aligned}
\dot{\overline{\bar{x}}}(t)= & \overline{\bar{x}}(t)\left[r-r_{1} \overline{\bar{x}}(t-\tau)-r_{2} \overline{\bar{u}}(t)-a_{1} \overline{\bar{y}}(t-\tau)\right] \\
\dot{\bar{u}}(t)= & \sigma \overline{\bar{x}}(t)-\sigma \overline{\bar{u}}(t) \\
\dot{\bar{y}}(t)= & \overline{\bar{y}}(t)\left[-r_{3}+a_{2} \overline{\bar{x}}(t-\tau)-r_{4} \overline{\bar{y}}(t-\tau)\right] \\
& -c \overline{\bar{y}}(t) \overline{\bar{y}}^{2}(t-\tau) .
\end{aligned}
$$

Let $\overline{\bar{x}}(t)=\left(r / r_{1}\right) \bar{x}(t), \overline{\bar{u}}(t)=\left(r / r_{1}\right) \bar{u}(t)$, and $\overline{\bar{y}}(t)=$ $\left(r_{3} / r_{4}\right) \bar{y}(t)$; by $(6)$,

$$
\begin{gathered}
\dot{\bar{x}}(t)=r \bar{x}(t)\left[1-\bar{x}(t-\tau)-\frac{r_{2}}{r_{1}} \bar{u}(t)-\frac{a_{1} r_{3}}{r r_{4}} \bar{y}(t-\tau)\right] \\
\dot{\bar{u}}(t)=\sigma \bar{x}(t)-\sigma \bar{u}(t) \\
\dot{\bar{y}}(t)=r_{3} \bar{y}(t)\left[-1+\frac{a_{2} r}{r_{1} r_{3}} \bar{x}(t-\tau)\right. \\
\left.-\bar{y}(t-\tau)-\frac{c r_{3}}{r_{4}^{2}} \bar{y}^{2}(t-\tau)\right] .
\end{gathered}
$$

Denote $a=r_{2} / r_{1}, b=a_{1} r_{3} / r r_{4}, c=a_{2} r / r_{1} r_{3}$, and $d=$ $c r_{3} / r_{4}^{2}$; from (7), it follows that

$$
\begin{aligned}
\dot{\bar{x}}(t)= & r \bar{x}(t)[1-\bar{x}(t-\tau)-a \bar{u}(t)-b \bar{y}(t-\tau)] \\
\dot{\bar{u}}(t)= & \sigma \bar{x}(t)-\sigma \bar{u}(t) \\
\dot{\bar{y}}(t)= & r_{3} \bar{y}(t) \\
& \times\left[-1+c \bar{x}(t-\tau)-\bar{y}(t-\tau)-d \bar{y}^{2}(t-\tau)\right] .
\end{aligned}
$$

\section{Main Results}

To ensure system (8) with positive equilibrium points, let us suppose system (8) with conditions

$$
\mathrm{G}_{1}: \sqrt{\Delta}-(1+a) d>0,(2+b)(1+a) d-\sqrt{\Delta}>0,
$$

where $\Delta=(1+a+b c)^{2}-4(1+a) d(1+a-c)$.

Clearly, $z^{*}=\left(x^{*}, u^{*}, y^{*}\right)$ is a positive equilibrium point of system (8) if and only if $z^{*}=\left(x^{*}, u^{*}, y^{*}\right)$ satisfies

$$
\begin{aligned}
& 0=1-x^{*}-a u^{*}-b y^{*} \\
& 0=b x^{*}-b u^{*} \\
& 0=-1+c x^{*}-y^{*}-d y^{* 2} .
\end{aligned}
$$

Obviously, under the assumption $G_{1}$, system (8) has unique positive equilibrium points $z^{*}=\left(x^{*}, u^{*}, y^{*}\right)$, where

$$
\begin{aligned}
& x^{*}=\frac{(2+b)(1+a) d-b \sqrt{\Delta}}{2(1+a)^{2} d} \\
& u^{*}=x^{*} \\
& y^{*}=\frac{\sqrt{\Delta}-(1+a+b c)}{2(1+a) d} .
\end{aligned}
$$

hen

$$
\text { Let } \bar{x}(t)=x(t)-x^{*}, \bar{u}(t)=u(t)-u^{*} \text {, and } \bar{y}(t)=y(t)-y^{*} \text {; }
$$

$$
\begin{aligned}
\dot{x}(t) & =r\left[x(t)+x^{*}\right][-x(t-\tau)-a u(t)-b y(t-\tau)] \\
\dot{u}(t) & =b x(t)-b u(t) \\
\dot{y}(t) & =r_{3}\left[y(t)+y^{*}\right] \\
& \times\left[c x(t-\tau)-\left(1+2 d y^{*}\right) y(t)-d y^{2}(t)\right] .
\end{aligned}
$$

Linearizing system (11) at $(0,0,0)$,

$$
\begin{aligned}
& \dot{x}(t)=r x^{*}[-x(t-\tau)-a u(t)-b y(t-\tau)] \\
& \dot{u}(t)=b x(t)-b u(t) \\
& \dot{y}(t)=r_{3} y^{*}\left[c x(t-\tau)-\left(1+2 d y^{*}\right) y(t)\right],
\end{aligned}
$$

then the characteristic equation of (12) is

$$
\begin{aligned}
\lambda^{3} & +d_{1} \lambda^{2}+d_{2} \lambda-d_{3}+\left(d_{4} \lambda^{2}+d_{5} \lambda-d_{6}\right) e^{-\lambda \tau} \\
& +\left(d_{7} \lambda+d_{8}\right) e^{-2 \lambda \tau}=0,
\end{aligned}
$$

where

$$
\begin{aligned}
& d_{1}=b-r_{3} y^{*}\left(1+2 d y^{*}\right), \\
& d_{2}=r a b x^{*}-r r_{3} x^{*} y^{*}\left(1+2 d y^{*}\right), \\
& d_{3}=r r_{3} a b x^{*} y^{*}\left(1+2 d y^{*}\right), \quad d_{4}=r x^{*}, \\
& d_{5}=r b x^{*}-r r_{3} x^{*} y^{*}\left(1+2 d y^{*}\right), \\
& d_{6}=r r_{3} b x^{*} y^{*}\left(1+2 d y^{*}\right), \\
& d_{7}=r r_{3} b c x^{*} y^{*}, \quad d_{8}=r r_{3} b^{2} c x^{*} y^{*} .
\end{aligned}
$$

By (13), we can get

$$
\begin{aligned}
& \left(\lambda^{3}+d_{1} \lambda^{2}+d_{2} \lambda-d_{3}\right) e^{\lambda \tau}+\left(d_{4} \lambda^{2}+d_{5} \lambda-d_{6}\right) \\
& +\left(d_{7} \lambda+d_{8}\right) e^{-\lambda \tau}=0 .
\end{aligned}
$$

Clearly, $i \omega(\omega>0)$ is a solution of (15) if and only if $\omega$ satisfies

$$
\begin{aligned}
& \left(-i \omega^{3}-d_{1} \omega^{2}+d_{2} i \omega-d_{3}\right) e^{i \omega}+\left(-d_{4} \omega^{2}+d_{5} i \omega-d_{6}\right) \\
& +\left(d_{7} i \omega+d_{8}\right) e^{-i \omega}=0 .
\end{aligned}
$$

Substituting $e^{i \omega}=\cos \omega \tau+i \sin \omega \tau, e^{-i \omega}=\cos \omega \tau-i \sin \omega \tau$ into (16), then

$$
\begin{aligned}
& \left(-i \omega^{3}-d_{1} \omega^{2}+d_{2} i \omega-d_{3}\right)(\cos \omega \tau+i \sin \omega \tau) \\
& +\left(-d_{4} \omega^{2}+d_{5} i \omega-d_{6}\right) \\
& +\left(d_{7} i \omega+d_{8}\right)(\cos \omega \tau-i \sin \omega \tau)=0 ;
\end{aligned}
$$


that is,

$$
\begin{aligned}
& {\left[\left(\omega^{3}-d_{2} \omega\right)-d_{7} \omega\right] \cos \omega \tau} \\
& \quad+\left[d_{8}+\left(d_{1} \omega+d_{3}\right)\right] \sin \omega \tau=d_{5} \omega, \\
& {\left[\left(\omega^{3}-d_{2} \omega\right)+d_{7} \omega\right] \sin \omega \tau} \\
& \quad+\left[d_{8}-\left(d_{1} \omega+d_{3}\right)\right] \cos \omega \tau=d_{4} \omega^{2}+d_{6} ;
\end{aligned}
$$

thus

$$
\begin{aligned}
\sin \omega \tau= & \left(d_{4} \omega^{5}+\left[d_{6}-d_{4}\left(d_{2}+d_{7}\right)\right] \omega^{3}+d_{1} d_{5} \omega^{2}\right. \\
& \left.+\left[d_{5}\left(d_{3}-d_{8}\right)-d_{6}\left(d_{2}+d_{7}\right)\right] \omega\right) \\
\times & \left(\omega^{6}-2 d_{2} \omega^{4}+\left(d_{1}^{2}+d_{2}^{2}-d_{7}^{2}\right) \omega^{2}\right. \\
& \left.+2 d_{1} d_{3} \omega+d_{3}^{2}-d_{8}^{2}\right)^{-1}, \\
\cos \omega \tau= & \left(d_{5} \omega^{4}-d_{1} d_{4} \omega^{3}+\left(d_{5} d_{7}-d_{2} d_{5}-d_{4} d_{8}-d_{3} d_{4}\right) \omega^{2}\right. \\
& \left.-d_{1} d_{6} \omega-\left(d_{3}+d_{8}\right) d_{6}\right) \\
\times & \left(\omega^{6}-2 d_{2} \omega^{4}+\left(d_{1}^{2}+d_{2}^{2}-d_{7}^{2}\right) \omega^{2}\right. \\
& \left.+2 d_{1} d_{3} \omega+d_{3}^{2}-d_{8}^{2}\right)^{-1} .
\end{aligned}
$$

Let

$$
\begin{aligned}
& e_{1}=-2 d_{2}, \quad e_{2}=d_{1}^{2}+d_{2}^{2}-d_{7}^{2}, \\
& e_{3}=2 d_{1} d_{3}, \quad e_{4}=d_{3}^{2}-d_{8}^{2}, \quad e_{5}=d_{4}, \\
& e_{6}=d_{6}-d_{4}\left(d_{2}+d_{7}\right), \quad e_{7}=d_{1} d_{5}, \\
& e_{8}=\left[d_{5}\left(d_{3}-d_{8}\right)-d_{6}\left(d_{2}+d_{7}\right)\right], \\
& e_{9}=d_{5}, \quad e_{10}=-d_{1} d_{4}, \\
& e_{11}=d_{5} d_{7}-d_{2} d_{5}-d_{4} d_{8}-d_{3} d_{4}, \\
& e_{12}=-d_{1} d_{6}, \quad e_{13}=-\left(d_{3}+d_{8}\right),
\end{aligned}
$$

so

$$
\begin{gathered}
\sin \omega \tau=\frac{e_{5} \omega^{5}+e_{6} \omega^{3}+e_{7} \omega^{2}+e_{8} \omega}{\omega^{6}+e_{1} \omega^{4}+e_{2} \omega^{2}+e_{3} \omega+e_{4}}, \\
\cos \omega \tau=\frac{e_{9} \omega^{4}+e_{10} \omega^{3}+e_{11} \omega^{2}+e_{12} \omega+e_{13}}{\omega^{6}+e_{1} \omega^{4}+e_{2} \omega^{2}+e_{3} \omega+e_{4}} .
\end{gathered}
$$

Combined with $\sin ^{2} \omega \tau+\cos ^{2} \omega \tau=1$, we get

$$
\begin{aligned}
\omega^{12}+ & f_{1} \omega^{10}+f_{2} \omega^{8}+f_{3} \omega^{7}+f_{4} \omega^{6}+f_{5} \omega^{5}+f_{6} \omega^{4}+f_{7} \omega^{3} \\
& +f_{8} \omega^{2}+f_{9} \omega+f_{10}=0
\end{aligned}
$$

where

$$
\begin{aligned}
& f_{1}=2 e_{1}-e_{5}^{2}, \quad f_{2}=2 e_{2}+e_{1}^{2}-2 e_{5} e_{6}-e_{9}^{2}, \\
& f_{3}=2\left(e_{3}-e_{5} e_{7}-e_{9} e_{10}\right), \\
& f_{4}=2 e_{4}+2 e_{1} e_{2}-e_{6}^{2}-e_{10}^{2}-2 e_{5} e_{8}-2 e_{9} e_{11}, \\
& f_{5}=2\left(e_{1} e_{3}-e_{6} e_{7}-e_{9} e_{12}-e_{10} e_{11}\right), \\
& f_{6}=e_{2}^{2}+2 e_{1} e_{4}-e_{7}^{2}-e_{11}^{2}-2 e_{6} e_{8}-2 e_{9} e_{13}-2 e_{10} e_{12}, \\
& f_{7}=2\left(e_{2} e_{3}-e_{7} e_{8}-e_{10} e_{13}-e_{11} e_{12}\right), \\
& f_{8}=e_{3}^{2}+2 e_{2} e_{4}-e_{8}^{2}-e_{12}^{2}-2 e_{11} e_{13}, \\
& f_{9}=2\left(e_{3} e_{4}-e_{12} e_{13}\right), \quad f_{10}=e_{4}^{2}-e_{13}^{2} .
\end{aligned}
$$

Let

$$
\begin{aligned}
G(\omega)= & \omega^{12}+f_{1} \omega^{10}+f_{2} \omega^{8}+f_{3} \omega^{7}+f_{4} \omega^{6}+f_{5} \omega^{5} \\
& +f_{6} \omega^{4}+f_{7} \omega^{3}+f_{8} \omega^{2}+f_{9} \omega+f_{10} .
\end{aligned}
$$

\section{Assume}

$\mathrm{G}_{2}$ : system (22) has at least one positive real solution. On the other hand,

$$
\cos \omega_{k} \tau=\frac{e_{9} \omega_{k}^{4}+e_{10} \omega_{k}^{3}+e_{11} \omega_{k}^{2}+e_{12} \omega_{k}+e_{13}}{\omega_{k}^{6}+e_{1} \omega_{k}^{4}+e_{2} \omega_{k}^{2}+e_{3} \omega_{k}+e_{4}} ;
$$

thus

$$
\begin{aligned}
\tau_{k}^{(j)}= & \frac{1}{\omega_{k}} \\
& \times\left\{\arccos \frac{e_{9} \omega_{k}^{4}+e_{10} \omega_{k}^{3}+e_{11} \omega_{k}^{2}+e_{12} \omega_{k}+e_{13}}{\omega_{k}^{6}+e_{1} \omega_{k}^{4}+e_{2} \omega_{k}^{2}+e_{3} \omega_{k}+e_{4}}+2 j \pi\right\},
\end{aligned}
$$

where $k=1, \ldots, 12, j=0,1, \ldots, \pm i \omega_{k}$. Define

$$
\tau_{0}=\tau_{k_{0}}^{(0)}=\min _{k \in\{1, \ldots, 12\}}\left\{\tau_{k}^{(0)}\right\}, \quad \omega_{0}=\omega_{k_{0}} .
$$

When $\tau=0$, we can obtain

$$
\lambda^{3}+\left(d_{1}+d_{4}\right) \lambda^{2}+\left(d_{2}+d_{5}+d_{7}\right) \lambda+d_{8}-d_{3}-d_{6}=0 \text {. }
$$

By Hurwitz criterion, it follows that the solutions of (28) are all of negative real parts when $\tau=0$, so the positive equilibrium point $Z^{*}$ is locally asymptotically stable.

In the following, we assume

$\mathrm{G}_{3}:\left.\operatorname{Re}(d \lambda / d \tau)\right|_{\tau=\tau_{0}} \neq 0$.

From (13)

$$
\begin{aligned}
\left(3 \lambda^{2}\right. & \left.+2 d_{1} \lambda+d_{2}\right) e^{\lambda \tau}+\left(\lambda^{3}+d_{1} \lambda^{2}+d_{2} \lambda-d_{3}\right) e^{\lambda \tau} \\
& \times\left(\lambda+\tau \frac{d \lambda}{d \tau}\right)+\left(2 d_{4} \lambda+d_{5}\right) \frac{d \lambda}{d \tau}+d_{7} e^{-\lambda \tau} \frac{d \lambda}{d \tau} \\
& -\left(d_{7} \lambda+d_{8}\right) e^{-\lambda \tau}\left(\lambda+\tau \frac{d \lambda}{d \tau}\right)=0
\end{aligned}
$$


therefore

$$
\begin{aligned}
& \frac{d \lambda}{d \tau} \\
& =\left(-\lambda\left(\lambda^{3}+d_{1} \lambda^{2}+d_{2} \lambda-d_{3}\right) e^{\lambda \tau}+\lambda\left(d_{7} \lambda+d_{8}\right) e^{-\lambda \tau}\right) \\
& \quad \times\left(\left(3 \lambda^{2}+2 d_{1} \lambda+d_{2}\right) e^{\lambda \tau}+\left(\lambda^{3}+d_{1} \lambda^{2}+d_{2} \lambda-d_{3}\right) \tau e^{\lambda \tau}\right. \\
& \left.\quad+2 d_{4} \lambda+d_{5}+d_{7} e^{-\lambda \tau}-\left(d_{7} \lambda+d_{8}\right) e^{-\lambda \tau}\right)^{-1}
\end{aligned}
$$

and then

$$
\begin{aligned}
\left(\frac{d \lambda}{d \tau}\right)^{-1} & \\
= & \frac{\left(3 \lambda^{2}+2 d_{1} \lambda+d_{2}\right) e^{\lambda \tau}+2 d_{4} \lambda+d_{5}+d_{7} e^{-\lambda \tau}}{-\lambda\left(\lambda^{3}+d_{1} \lambda^{2}+d_{2} \lambda-d_{3}\right) e^{\lambda \tau}+\lambda\left(d_{7} \lambda+d_{8}\right) e^{-\lambda \tau}} \\
& -\frac{\tau}{\lambda} \\
= & \frac{\left(3 \lambda^{2}+2 d_{1} \lambda+d_{2}\right) e^{\lambda \tau}+2 d_{4} \lambda+d_{5}+d_{7} e^{-\lambda \tau}}{d_{4} \lambda^{3}+d_{5} \lambda^{2}-d_{6} \lambda+2 \lambda\left(d_{7} \lambda+d_{8}\right) e^{-\lambda \tau}}-\frac{\tau}{\lambda} \\
= & \frac{\left(-3 \omega^{2}+2 d_{1} i \omega+d_{2}\right) e^{\lambda \tau}+2 d_{4} i \omega+d_{5}+d_{7} e^{-\lambda \tau}}{-d_{4} i \omega^{3}-d_{5} \omega^{2}-d_{6} i \omega+2 i \omega\left(d_{7} i \omega+d_{8}\right) e^{-\lambda \tau}} \\
& -\frac{\tau}{i \omega} .
\end{aligned}
$$

Substituting $e^{i \omega}=\cos \omega \tau+i \sin \omega \tau, e^{-i \omega}=\cos \omega \tau-i \sin \omega \tau$ into (31), we get

$$
\begin{aligned}
& \left(\frac{d \lambda}{d \tau}\right)^{-1} \\
& =\left(\left(-3 \omega^{2}+2 d_{1} i \omega+d_{2}\right)(\cos \omega \tau+i \sin \omega \tau)\right. \\
& \left.\quad+2 d_{4} i \omega+d_{5}+d_{7}(\cos \omega \tau-i \sin \omega \tau)\right) \\
& \times\left(-d_{4} i \omega^{3}-d_{5} \omega^{2}-d_{6} i \omega\right. \\
& \left.\quad+2 i \omega\left(d_{7} i \omega+d_{8}\right)(\cos \omega \tau-i \sin \omega \tau)\right)^{-1}-\frac{\tau}{i \omega} \\
& =\left(\left(-3 \omega^{2}+d_{2}+d_{7}\right) \cos \omega \tau-2 d_{1} \omega \sin \omega \tau+d_{5}\right) \\
& \times\left(\left(-d_{5} \omega^{2}-2 d_{7} \omega^{2} \cos \omega \tau+2 d_{8} \omega \sin \omega \tau\right)\right. \\
& \left.\quad+i\left(-d_{4} \omega^{3}-d_{6} \omega+2 d_{8} \omega \cos \omega \tau+2 d_{7} \omega^{2} \sin \omega \tau\right)\right)^{-1} \\
& +\left(i\left[2 d_{1} \omega \cos \omega \tau+\left(-3 \omega^{2}+d_{2}-d_{7}\right) \sin \omega \tau+2 d_{4} \omega\right]\right) \\
& \times\left(\left(-d_{5} \omega^{2}-2 d_{7} \omega^{2} \cos \omega \tau+2 d_{8} \omega \sin \omega \tau\right)\right. \\
& \left.\quad+i\left(-d_{4} \omega^{3}-d_{6} \omega+2 d_{8} \omega \cos \omega \tau+2 d_{7} \omega^{2} \sin \omega \tau\right)\right)^{-1} \\
& +i \omega
\end{aligned}
$$

Let

$$
\begin{aligned}
& Q=\left.-d_{5} \omega^{2}-2 d_{7} \omega^{2} \cos \omega \tau+2 d_{8} \omega \sin \omega \tau\right)^{2} \\
&+\left(-d_{4} \omega^{3}-d_{6} \omega+2 d_{8} \omega \cos \omega \tau+2 d_{7} \omega^{2} \sin \omega \tau\right)^{2} \\
&>0 \\
& Q \operatorname{Re}\left(\frac{d \lambda}{d \tau}\right)^{-1} \\
&=\left[\left(-3 \omega^{2}+d_{2}+d_{7}\right) \cos \omega \tau-2 d_{1} \omega \sin \omega \tau+d_{5}\right] \\
& \quad \times\left[\left(-d_{5} \omega^{2}-2 d_{7} \omega^{2} \cos \omega \tau+2 d_{8} \omega \sin \omega \tau\right)\right] \\
&+ {\left[2 d_{1} \omega \cos \omega \tau+\left(-3 \omega^{2}+d_{2}-d_{7}\right) \sin \omega \tau+2 d_{4} \omega\right] } \\
& \times\left[-d_{4} \omega^{3}-d_{6} \omega+2 d_{8} \omega \cos \omega \tau+2 d_{7} \omega^{2} \sin \omega \tau\right]
\end{aligned}
$$

Combined with

$$
\operatorname{sign}\left\{\left.\operatorname{Re}\left(\frac{d \lambda}{d \tau}\right)\right|_{\tau=\tau_{0}}\right\}=\operatorname{sign}\left\{\left.\operatorname{Re}\left(\frac{d \lambda}{d \tau}\right)\right|_{\tau=\tau_{0}} ^{-1}\right\},
$$

then we can derive the following theorem.

Theorem 1. If assumptions $G_{2}, G_{3}$ hold, then

(i) when $\tau \in\left[0, \tau_{0}\right)$, the positive equilibrium point $z^{*}\left(x^{*}, u^{*}, y^{*}\right)$ of system (11) is asymptotically stable;

(ii) when $\tau=\tau_{0}$, the region near the positive equilibrium point $z^{*}\left(x^{*}, u^{*}, y^{*}\right)$ of system (11) can appear Hopf bifurcation.

\section{Conclusion}

As we know, time delay has a strong impact on the dynamic evolution of a population, which could bring a switch in the stability of equilibria and induce various oscillations and periodic solutions. However, these effects would induce ecosystem unbalance and even biological disaster. In order to maintain the sustainable development of the biological resources, we investigate a predator-prey model with delays. In this paper, on the basis of Lotka-Volterra predator-prey system, a general class of predator-prey models with distributed delays and competition term is formulated and studied. Some sufficient conditions in terms of algebraic criterion are obtained, in order to achieve stability and Hopf bifurcation. These theoretical analyses can characterize the fundamental properties of ecological system and provide convenience for applications. By using the same method, similar results may be obtained for the predator-prey model with functional response which describes prey eaten per predator per unit of time.

In the future, we will investigate the direction of Hopf bifurcation and the stability of the bifurcating periodic solutions based on the normal form approach theory and center manifold theory introduced by Hassard et al. [11]. 


\section{Conflict of Interests}

The author declares that there is no conflict of interests regarding the publication of this paper.

\section{References}

[1] L. Wu, Z. Feng, and J. Lam, "Stability and synchronization of discrete-time neural networks with switching parameters and time-varying delays," IEEE Transactions on Neural Networks and Learning Systems, vol. 24, no. 12, pp. 1957-1972, 2013.

[2] L. Wu, Z. Feng, and W. X. Zheng, "Exponential stability analysis for delayed neural networks with switching parameters: average dwell time approach," IEEE Transactions on Neural Networks, vol. 21, no. 9, pp. 1396-1407, 2010.

[3] H. Li, H. Gao, and P. Shi, "New passivity analysis for neural networks with discrete and distributed delays," IEEE Transactions on Neural Networks, vol. 21, no. 11, pp. 1842-1847, 2010.

[4] H. Li, B. Chen, Q. Zhou, and W. Qian, "Robust stability for uncertain delayed fuzzy Hopfield neural networks with Markovian jumping parameters," IEEE Transactions on Systems, Man, and Cybernetics B: Cybernetics, vol. 39, no. 1, pp. 94-102, 2009.

[5] R. M. May, "Time delay versus stability in population models with two and three trophic levels," Ecology, vol. 54, no. 2, pp. 315-325, 1973.

[6] J. Hale, Theory of Functional Differential Equations, Springer, New York, NY, USA, 1977.

[7] G. Zhang, B. Chen, L. Zhu, and Y. Shen, "Hopf bifurcation for a differential-algebraic biological economic system with time delay," Applied Mathematics and Computation, vol. 218, no. 15, pp. 7717-7726, 2012.

[8] B. Chen and J. Chen, "Bifurcation and chaotic behavior of a discrete singular biological economic system," Applied Mathematics and Computation, vol. 219, no. 5, pp. 2371-2386, 2012.

[9] Y. Song and J. Wei, "Local Hopf bifurcation and global periodic solutions in a delayed predator-prey system," Journal of Mathematical Analysis and Applications, vol. 301, no. 1, pp. 1-21, 2005.

[10] X.-P. Yan and W.-T. Li, "Hopf bifurcation and global periodic solutions in a delayed predator-prey system," Applied Mathematics and Computation, vol. 177, no. 1, pp. 427-445, 2006.

[11] B. D. Hassard, N. D. Kazarinoff, and Y. H. Wan, Theory and Applications of Hopf Bifurcation, vol. 41, Cambridge University Press, Cambridge, UK, 1981. 




Advances in

Operations Research

mansans

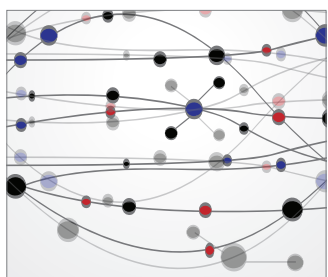

The Scientific World Journal



Submit your manuscripts at http://www.hindawi.com


Journal of

Function Spaces



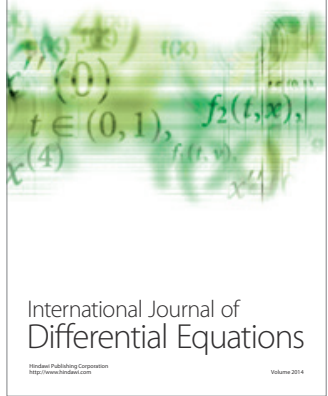
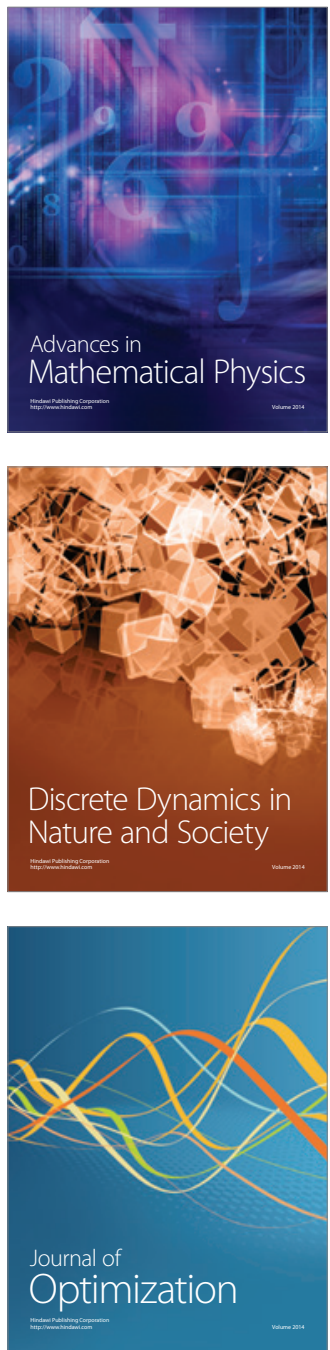